\title{
Multi-component, Self-assembled, Functional Soft Materials
}

\author{
V. S. Sajisha and Uday Maitra*
}

\begin{abstract}
Supramolecular chemistry is an emerging tool for devising materials that can perform specified functions. The self-assembly of facially amphiphilic bile acid molecules has been extensively utilized for the development of functional soft materials. Supramolecular hydrogels derived from the bile acid backbone act as useful templates for the intercalation of multiple components. Based on this, synthesis of gel-nanoparticle hybrid materials, photoluminescent coating materials, development of a new enzyme assay technique, etc. were achieved in the author's laboratory. The present account highlights some of these achievements.
\end{abstract}

Keywords: Enzyme assay · Functional soft materials · Gel-nanoparticle hybrid materials · Lanthanide luminescence $\cdot$ Self-assembly $\cdot$ Supramolecular gels
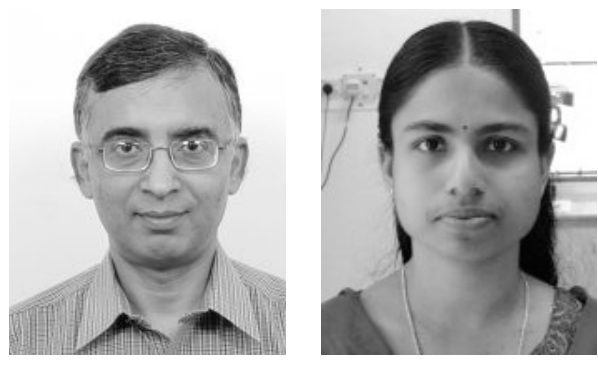

Uday Maitra had his early education at Presidency College, Calcutta and at IIT Kanpur. He did his $\mathrm{PhD}$ work at Columbia University with Prof. Ronald Breslow, followed by postdoctoral work at the University of California, Berkeley, with Prof. Paul A. Bartlett. He has been at the Indian Institute of Science, Bangalore since 1989 where he is currently a Professor. His research interests include bile acid chemistry, self-assembled systems and functional molecular gels. He is also greatly interested in chemical education and innovative methods of teaching.

Sajisha V.S. received her Masters degree in Chemistry from the Cochin University of Science and Technology, Kerala. She did her $\mathrm{PhD}$ under the supervision of Dr. K. V. Radhakrishnan at NIIST, Thiruvananthapuram. After a postdoctoral tenure with Prof. Hidehiro Sakurai at the Institute for Molecular Sciences, Japan she joined Prof. Uday Maitra's group at the Department of Organic Chemistry, Indian Institute of Science, Bangalore as a D.S. Kothari postdoctoral fellow in 2011. Her current research is focused on bile acid-

${ }^{*}$ Correspondence: Prof. U. Maitra Department of Organic Chemistry Indian Institute of Science

Bangalore 560012, India

E-mail: maitra@orgchem.iisc.ernet.in based supramolecular gels and hybrid nanomaterials.

\section{Introduction}

Functional soft materials continue to attract chemists and biologists owing to their stimuli responsive nature, reversibility and self-healing. ${ }^{[1]}$ There has been considerable interest in devising such materials in the last two decades due to their wide range of applications in tissue engineering, regenerative medicine, optoelectronics etc. [2] Self-assembly of various organic and inorganic components is a practical tool for building soft materials which can perform specified functions. But the fundamental challenge is how to "controllably organize' multiple components in engineering robust materials. All attempts in this area are inspired by the Nature's way of selfassembling various complex functionalities to generate functional materials with optimized properties. This brief review highlights progress made in the author's laboratory towards the development of bile acid derived functional gels.

Supramolecular gels have emerged as a new class of soft materials amenable to a variety of synthetic manipulations to achieve a variety of functions. Gels are defined as viscoelastic, solid-like materials. The selfassembly of small organic molecules to form three-dimensional networks creates matrices that can hold solvent molecules, thereby forming gels. The gel network is often made up of nanofibres which are held together by non-covalent interactions such as hydrogen bonding, hydrophobic interactions, $\pi$-stacking etc. There are different classes of gels based on their constitution, the medium they encompass and the crosslinking present (Fig. 1). ${ }^{[3]}$
Steroids, peptides, carbohydrates etc. are well known low molecular weight gelators (LMWG). Bile salts are facially amphiphilic molecules that self-assemble in aqueous media. Our group has extensively studied the self-assembly of a variety of bile-acid derivatives. We have reported bile acid-based sensors for cations and anions, ${ }^{[4]}$ light harvesting dendrimers ${ }^{[5]}$ and other dendritic structures that can solubilize cholesterol ${ }^{[6]}$ and selectively extract dye molecules ${ }^{[7]}$ from a mixture. Our current interest is on the supramolecular organo-, hydro- and metallogels derived from the bile acid backbone. The present account focuses mainly on the functional materials synthesized in bile acid-based supramolecular hydrogel matrices.

\section{A Tripodal Cholamide as a Super Hydrogelator}

The serendipitous discovery of a tripodal cholamide derivative as a super hy-

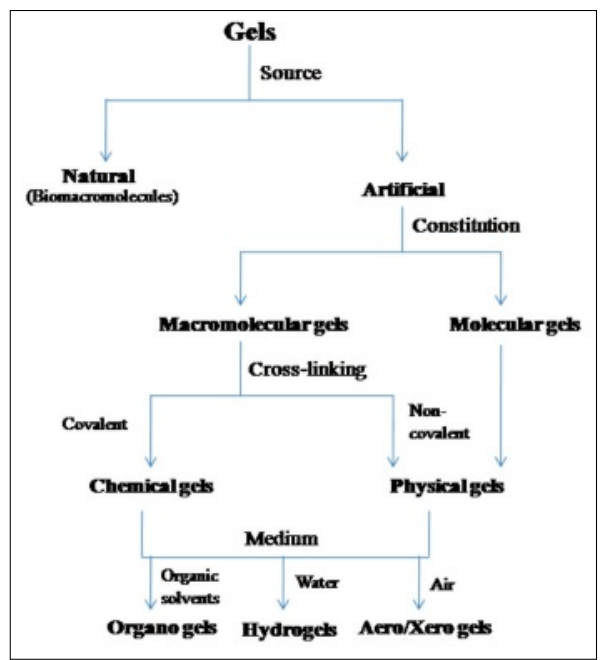

Fig. 1. Classification of gels. 


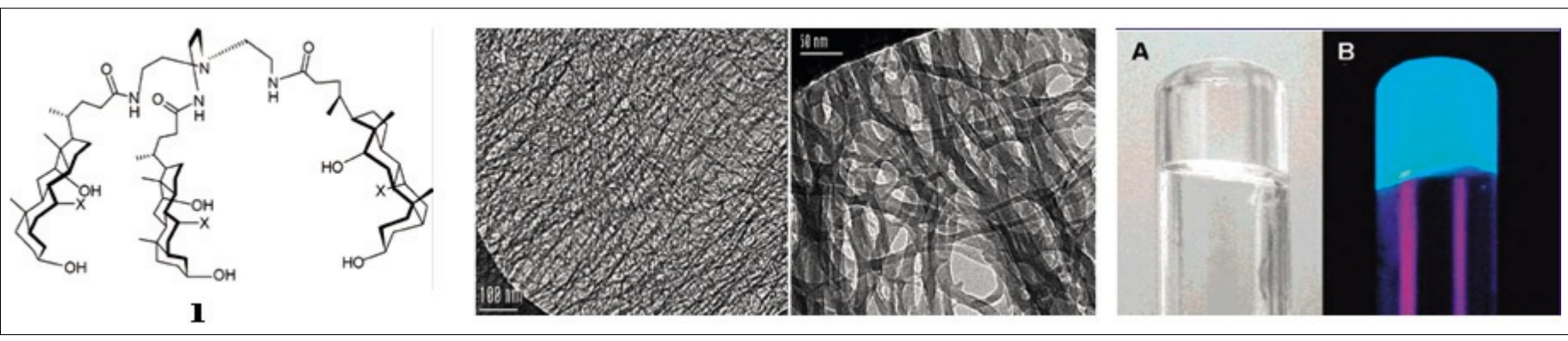

Fig. 2. Tripodal cholamide, cryoTEM pictures and ANS fluorescence in the gel. Reprinted with permission from ref. [8c]. Copyright 2004 American Chemical Society.

drogelator ushered in new research areas in our group. This nona-hydroxy cholic acid derivative was found to be an efficient gelator of aqueous acids. The fibrillar morphology of the gel was clearly observed from cryo-transmission electron microscopy (TEM) imaging. The presence of strong hydrophobic pockets in this gel was demonstrated by the use of 8-anilino1-naphthalene sulfonic acid (ANS) as a polarity sensitive fluorescence probe (Fig. 2). Other organic dye molecules were also doped in the gel phase and the rotational dynamics of these molecules were studied in detail. A (reversible) thermochromic gel was devised by the addition of the sodium salt of bromophenol blue to a solution of the gelator in $\mathrm{AcOH} /$ water, showing a yellow colour in the sol state which changed to green upon gelation. ${ }^{[8]}$

\section{A Thermochromic System Based on Phosphonobile Acids} tors synthesized in our laboratory is the phosphonobile salts. ${ }^{[9]}$ A thermochromic gel system was developed using these derivatives. Congo red, which shows a $\mathrm{pH}$ -
Another class of $\mathrm{pH}$-dependent gela-

dependent colour change, was intercalated with the phosphonodeoxycholate which formed gels in the $\mathrm{pH}$ range of 3.1-3.4. This formed a thermochromic system where the gel and sol appeared violet and magenta, respectively (Fig. 3). This discovery further emphasized the utility of these systems as novel functional materials.

\section{Gel-Nanoparticle Hybrid Materials}

Stabilization and surface functionalization of metal nanoparticles is an active research area owing to their unusual catalytic, magnetic, optical and other properties $^{[10]}$ which enable them to be used in several fields like biomedical science, catalysis and sensing.

We have designed gel-nanoparticle hybrid materials using different bile-acid derivatives. A special class of facially amphiphilic thiols were used to cap the in situ-generated gold nanoparticles by the $\mathrm{NaBH}_{4}$ mediated reduction of $\mathrm{HAuCl}_{4}$. These nanoparticles were characterized by the typical surface plasmon resonance band at $520 \mathrm{~nm}$. TEM analysis showed that spherical nanoparticles $(1.5-3.5 \mathrm{~nm})$ were

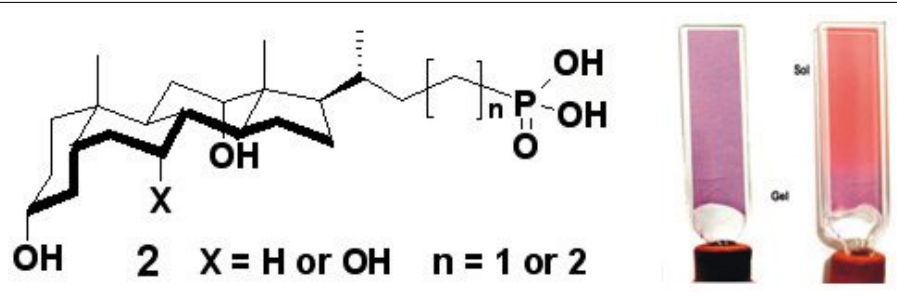

Fig. 3. Phosphonobile acids and the thermochromism shown by a phosphonodeoxycholic acid based gel. Reproduced from ref. [9] by permission of The Royal Society of Chemistry.

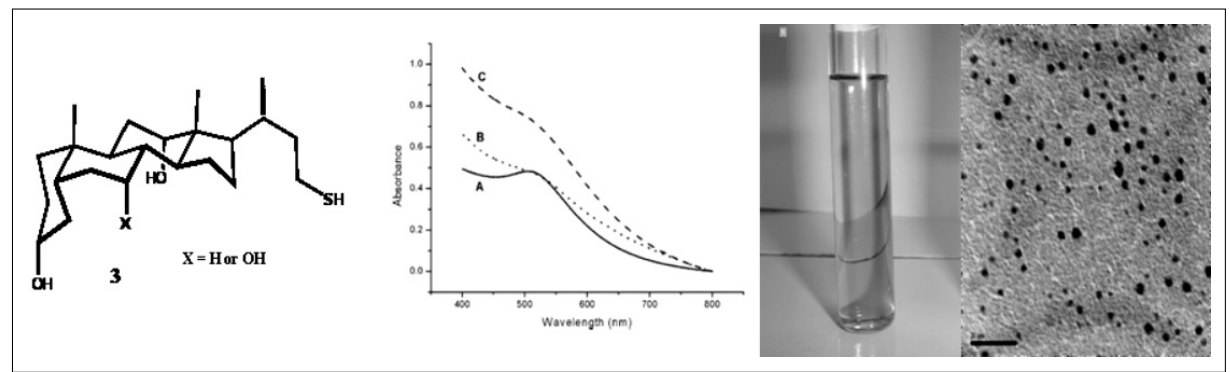

Fig. 4. Bile acid derived thiol capped gold nanopartles, absorption spectrum and TEM image. Reprinted with permission from ref. [11]. Copyright 2006 American Chemical Society. formed depending on the specific thiol derivative used (Fig. 4).

These steroid capped gold nanoparticles were then immobilized in the hydrogel derived from tripodal cholamide. ${ }^{[11]}$ The nanoparticles were found to be stable in the gel matrix without affecting the gel morphology. TEM imaging showed a regular arrangement of these nanoparticles on the gel fibres (Fig. 5). Importantly, the nanoparticles were shown to be stable in the medium (aq. acetic acid) only in the gel matrix. Conversion of the gel to the sol resulted in the precipitation of the nanoparticles.

\section{Synthesis of Nanoparticles in Metal Cholate Hydrogels}

The first report on the formation of a metal cholate hydrogel was published in 1913 by Schryver, which he termed 'clot' formation. He showed that $\mathrm{Ca}$ (II) triggered the gelation of an aqueous solution of sodium cholate. ${ }^{[12]}$ It was subsequently found that the calcium cholate gel is a self-assembled network of one-dimensional nanohelices. ${ }^{[13]}$ Gelation of sodium cholate solution effected by other divalent and monovalent metal ions (Fig. 6) was reported from our group. ${ }^{[14]}$

Electron microscopy studies showed that the gels were made up of three-dimensional fibrillar networks. Some interesting observations were made during the TEM analysis of calcium cholate gels made from calcium nitrate as well as calcium chloride. The gel prepared using calcium chloride did not show any helical nano-fibres whereas the one with calcium nitrate showed lefthanded helical fibres with 60-70 nm pitch. This unusual anion dependence of the gel morphology is being investigated (Fig. 7).

We were intrigued by the possibility of using the calcium cholate nanofibres for the synthesis and stabilization of organicinorganic composite materials. Direct exposure of the $\mathrm{Co}^{2+}, \mathrm{Zn}^{2+}, \mathrm{Cu}^{2+}, \mathrm{Cd}^{2+}$ cholate gels to $\mathrm{H}_{2} \mathrm{~S}$ led to the precipitation of the corresponding sulfides with the collapse of the gel network. We, therefore, planned to incorporate these metal ions into the rigid 


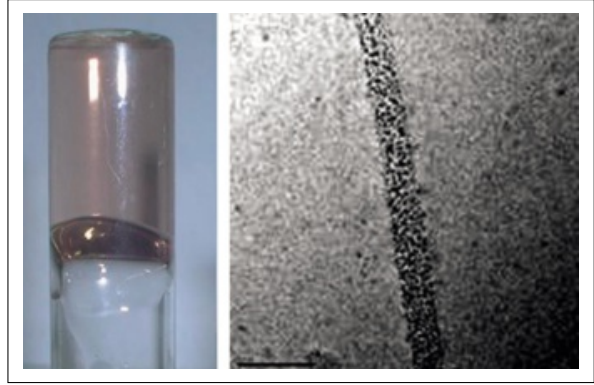

Fig. 5. Thiol capped gold nanoparticles immobilized in gel medium and the TEM image. Reprinted with permission from ref. [11]. Copyright 2006 American Chemical Society.

calcium cholate hydrogel matrix by partial doping, with the idea that subsequent exposure to aq. $\mathrm{Na}_{2} \mathrm{~S}$ would result in the formation of corresponding metal sulphide nanoparticles embedded on the intact $\mathrm{Ca}$ cholate gel fibre. Execution of this idea led to the synthesis of several metal sulphide nanoparticle-gel composites (Fig. 8).

$\mathrm{CdS}$ nanoparticles were synthesized by exposing the $\mathrm{Cd}$ (II) doped calcium cholate gel to the sulphiding agent $\mathrm{Na}_{2} \mathrm{~S}$. The TEM imaging and analysis of this material showed that the nanoparticles having zinc blend structure with 3-6 nm were formed inside the gel fibres (Fig. 9).

Gold and silver nanoparticles were also synthesized in the calcium cholate gel matrix by the slow diffusion of sodium cyanoborohydride into the gel doped with gold(III) chloride or silver nitrate, respectively. Absorption spectra of both nanoparticles (NPs) showed the corresponding surface plasmon resonance (SPR) peaks (Fig. 10).

These results indicate the effective utilization of the calcium cholate gel matrix as a template for the synthesis of metal sulfides. This strategy appears to be a general way to embed inorganic nanostructures in a hydrogel matrix. These stable nano-materials can be used for specified applications.

\section{Lanthanide Cholate Hydrogels as Functional Materials}

The study of lanthanide ions has been of great interest due to their extraordinary luminescent properties. They show environment independent sharp line-like emission with long lifetimes which make them good candidates for optical imaging and sensing. ${ }^{[15]}$ However, the aqueous lanthanide ions possess low molar extinction coefficients due to the Laporte forbidden $\mathrm{f}-\mathrm{f}$ transitions. Thus, for analytical purposes lanthanide ions are typically complexed with suitable ligands to give sufficiently strong emission. ${ }^{[16]}$ This strategy involves tedious synthetic steps and the efficiency

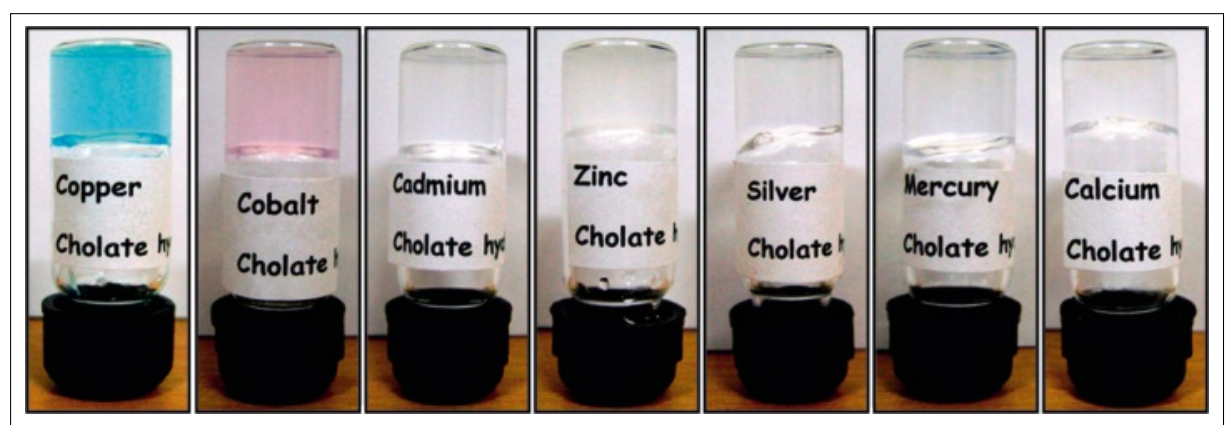

Fig. 6. Metal cholate hydrogels. Reproduced from ref. [14] by permission of The Royal Society of Chemistry.
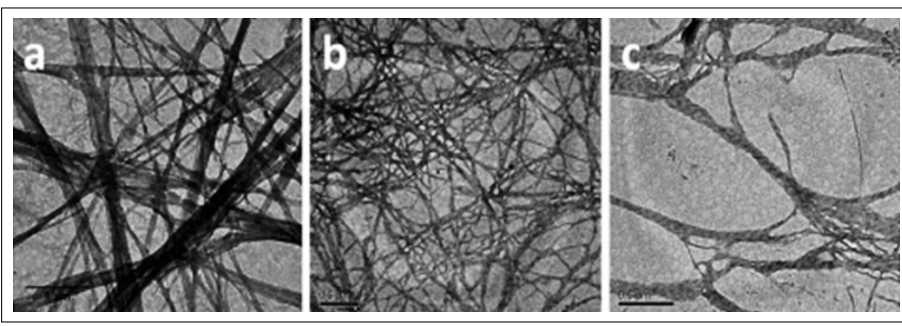

Fig. 7. TEM images of (a) $\mathrm{CaCl}_{2}$ derived hydrogel (b) \& (c) $\mathrm{Ca}\left(\mathrm{NO}_{3}\right)_{2}$ derived hydrogel. Reproduced from ref. [14] by permission of The Royal Society of Chemistry.

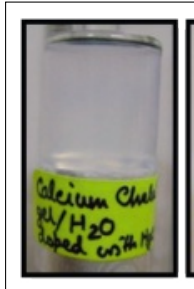

a

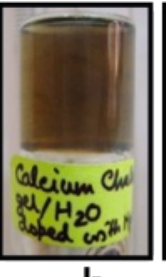

b

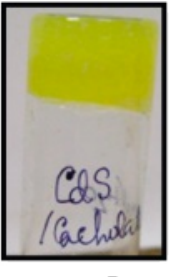

C

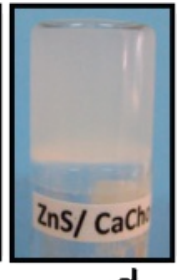

d

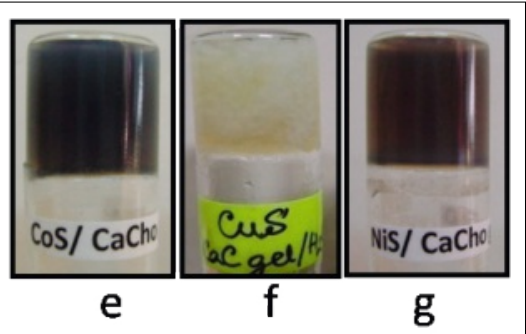

$\mathrm{g}$

Fig. 8. Various metal sulfide nanoparticles synthesized in calcium cholate hydrogel. Reproduced from ref. [14] by permission of The Royal Society of Chemistry.

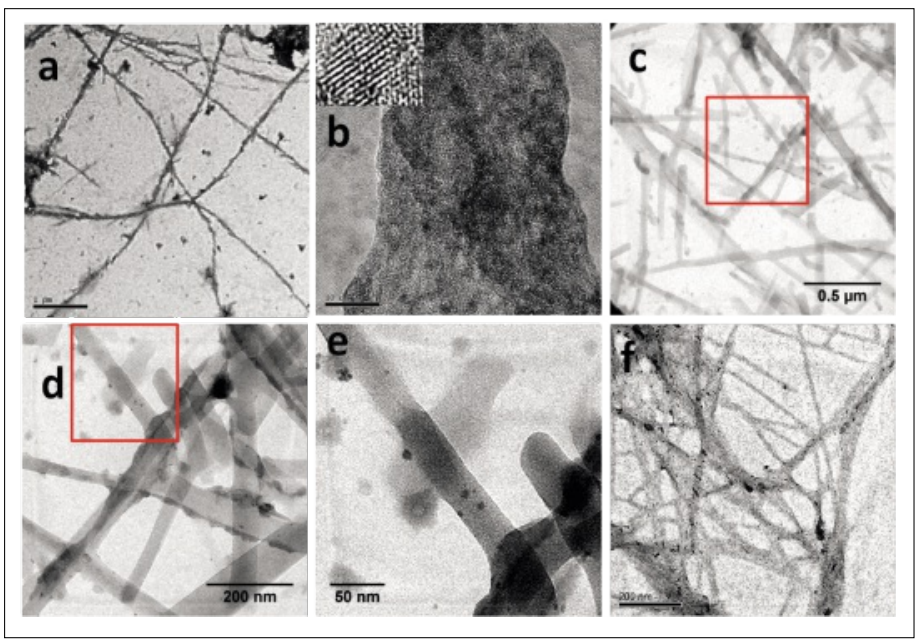

Fig. 9. TEM images of ( $a$ and $b$ ) CdS-calcium cholate hybrid gel (scale bars $1 \mathrm{~mm}$ and $10 \mathrm{~nm}$ respectively; inset: HRTEM of a single CdS nanoparticle present inside the fibre); (c-e) ZnS-calcium cholate hydrogel (the red box area has been zoomed in the corresponding images from (c-e), scale bars $0.5 \mathrm{~mm}, 200 \mathrm{~nm}$ and $50 \mathrm{~nm}$ respectively) and (f) HgS-calcium cholate hybrid gels (scale bar $200 \mathrm{~nm}$ ). Reproduced with permission from ref. [14]. () 2012 Royal Society of Chemistry.

of the ligands is somewhat unpredictable. A strategy developed many decades ago was the compartmentalization of lanthanide ions and sensitizers using an anionic micelle ${ }^{[17]}$ to enhance the lanthanide luminescence. The solid-state luminescence of lanthanide ions and their complexes was used in optoelectronic applications by embedding them in appropriate matrices. ${ }^{[18]}$

We presumed that a self-assembled lanthanide derived gel doped with a suitable sensitizer might show enhanced lan- 


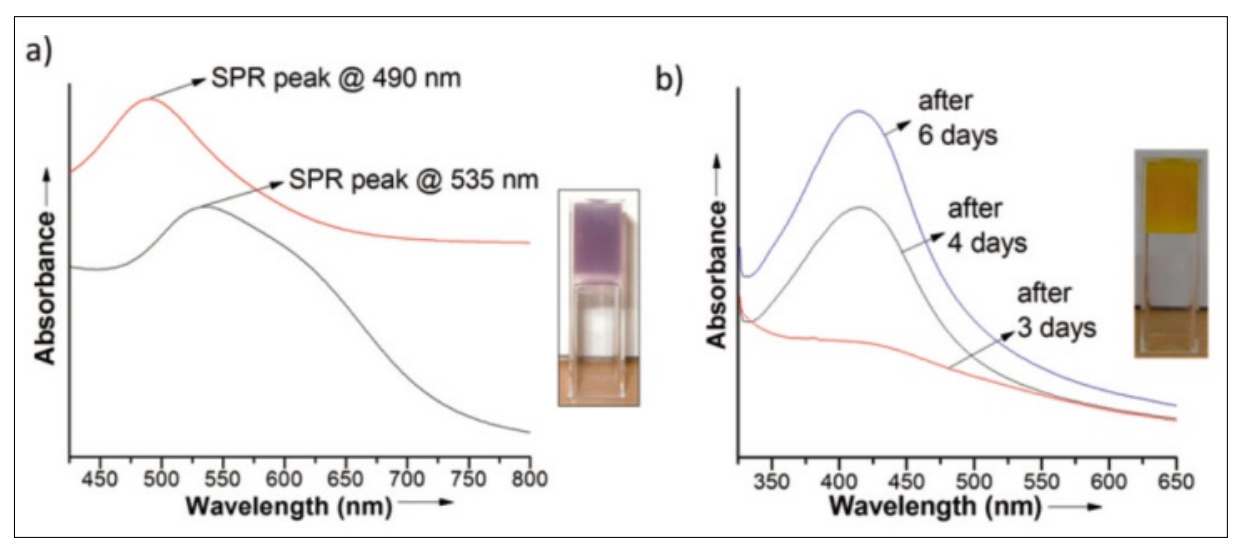

Fig. 10. Absorption spectrum of (a) gold and (b) silver nanoparticles synthesized in calcium cholate hydrogel. Reproduced from ref. [14] by permission of The Royal Society of Chemistry.

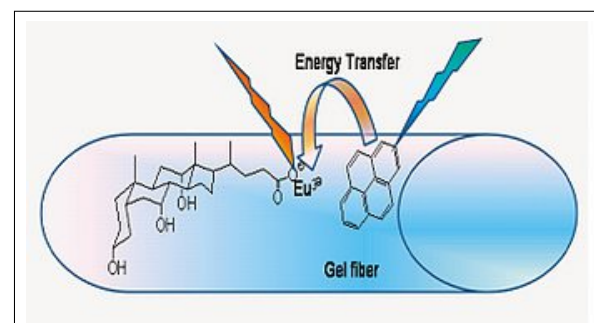

(a)

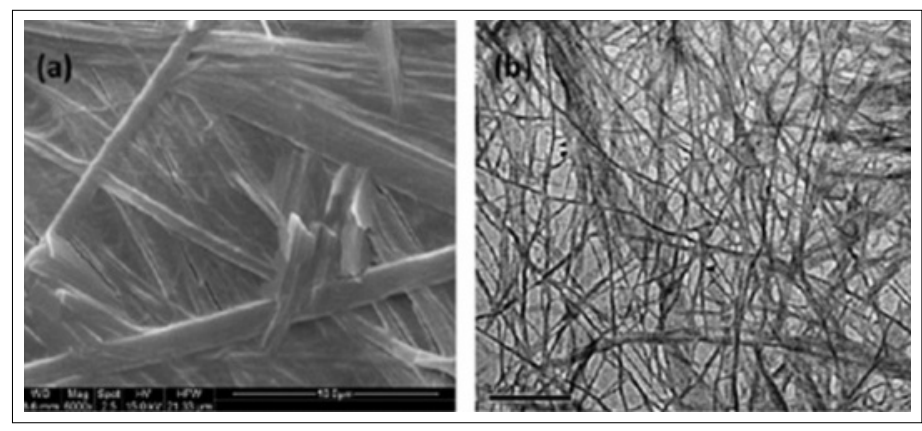

Fig. 11. (a) Eu(III) sensitization by pyrene (b) Eu cholate gel (c) Eu cholate gel with pyrene showing pink emission. Reproduced from ref [20] by permission of The Royal Society of Chemistry.

Fig. 13. SEM and TEM pictures of Eu cholate hydrogel. Reproduced from ref. [20] by permission of The Royal Society of Chemistry.

thanide luminescence which may be used for developing functional materials. Our previous studies showed that divalent and monovalent metal ions are capable of gelling aqueous solution of sodium cholate. These results suggested that we could incorporate lanthanides as an integral component of the gel system. Even though lanthanide-doped supramolecular gels were known, no significant enhancement of the luminescence was reported. [19] The first example of europium sensitization in a europium cholate supramolecular gel doped with pyrene as a sensitizer was recently reported by us (Fig. 11). Pyrene has no functionalities that can coordinate with europium. But in the supramolecular gel matrix all the components are immobilized, with pyrene in the close vicinity of Eu(III) which helps the energy transfer from pyrene to $\mathrm{Eu}(\mathrm{III}) .{ }^{[20]}$

The photoluminescence spectra suggested that there was significant energy transfer from pyrene to Eu(III) resulting in strong red emission. As little as $6 \mu \mathrm{M}$ of doped pyrene led to a 10-fold increase in the Eu(III) emission. This was unique for the cholate gel matrix as no emission enhancement in the sodium dodecyl sulfate (SDS) micelle with same amount of pyrene was observed (Fig. 12).

Scanning electron microscopy (SEM) analysis of the Eu cholate gel showed flat tape-like structures, whereas TEM analysis showed fibrous structures of diameter 20-30 nm (Fig. 13).

Gelation of aqueous sodium cholate solution was found to be general for all the lanthanide ions tested, with $5 \mathrm{mM}$ lanthanide and $15 \mathrm{mM}$ sodium cholate giving strong and transparent gels. Our next attempt was to study the sensitization of other lanthanides in a hydrogel matrix. 2,3-Dihydroxynaphthalene (DHN) was found to sensitize $\mathrm{Tb}$ (III) at micromolar concentrations in the gel to produce green emission $(10 \mu \mathrm{M}$ of DHN giving 7.5-fold emission enhancement). As in the case of $\mathrm{Eu}(\mathrm{III})$ sensitization using pyrene, DHN also did not show significant sensitization

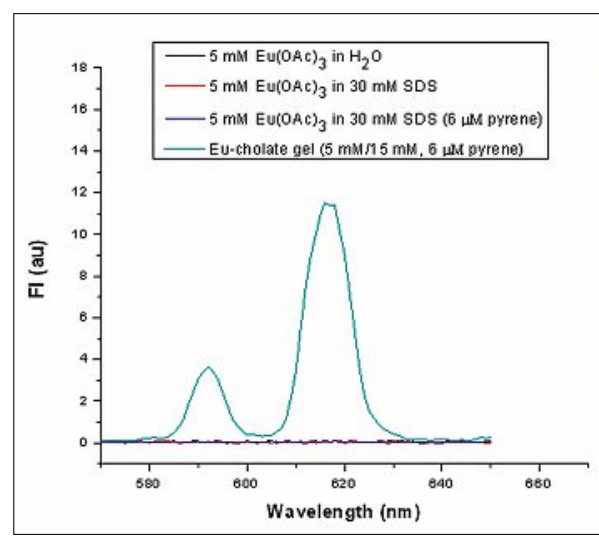

Fig. 12. Time delayed emission spectrum of Eu(III) in different matrices. Reproduced from ref. [20] by permission of The Royal Society of Chemistry.

of $\mathrm{Tb}$ (III) in SDS micelles (Fig. 14). The choice of the chromophore was important for sensitization as we observed that DHN sensitized Tb(III) but not Eu(III), while pyrene sensitized Eu(III) but not Tb(III).

$\mathrm{Tb}$ (III) to Eu(III) energy transfer in their mixed complexes, ${ }^{[21]}$ in co-ordination polymers ${ }^{[22]}$ and lanthanide doped polymeric gels, ${ }^{[23]}$ in a zeolite cavity, ${ }^{[24]}$ etc. has been reported in the literature. We explored the possibility of energy transfer from $\mathrm{Tb}$ (III) to Eu(III) in their mixed hydrogels. Thus varying amounts of $\mathrm{Tb}$ (III) and Eu(III) were used to make the cholate hydrogel where the total concentration of the lanthanide ions used remained $5 \mathrm{mM}$. When this mixed gel was doped with DHN it was observed that DHN sensitized $\mathrm{Tb}$ (III) and there was an efficient energy transfer from $\mathrm{Tb}$ (III) to $\mathrm{Eu}(\mathrm{III})$. The gel simultaneously acted as a green as well as a red emitter. By carefully tuning the ratios of both lanthanides, we were able to generate yellow and orange emissions. A mixed gel containing $4.9 \mathrm{mM}$ of $\mathrm{Tb}(\mathrm{III})$ and $0.1 \mathrm{mM}$ of $\mathrm{Eu}(\mathrm{III})$ showed yellow emission while reddish pink emission was obtained from a gel having $4 \mathrm{mM}$ of $\mathrm{Tb}$ (III) and $1 \mathrm{mM}$ of Eu(III) (Fig. 15).

As mentioned earlier, strong emission of lanthanides in the solid state is highly useful for optoelectronic applications. So we made the xerogels by freeze drying the highly emissive sensitizer-doped lanthanide cholate gels and investigated emission in the solid state. The xerogels showed relatively higher emission intensity compared to the hydrogels, probably due to the absence of hydroxyl quenching. The enhancements in the $\mathrm{Eu}(\mathrm{III})$ and $\mathrm{Tb}$ (III) emissions in the doped xerogels compared to the undoped ones were similar to that observed in the hydrogels. Energy transfer from $\mathrm{Tb}$ (III) to Eu(III) was also observed in the xerogel (Fig. 16).

The xerogels seemed to be interesting for developing photoluminescent coating materials. These xerogels were dispersed in 


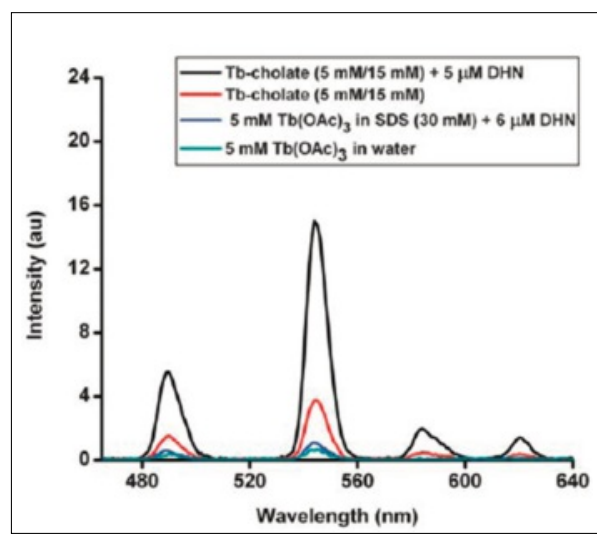

Fig. 14. Time delayed emission spectrum of $\mathrm{Tb}$ (III) in different matrices. Reproduced from ref. [25] by permission of The Royal Society of Chemistry.

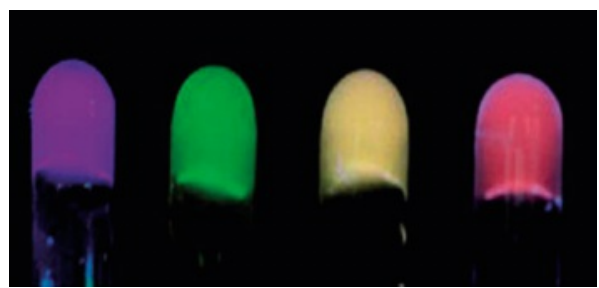

Fig. 15. Photographs of lanthanide (III) cholate gels under UV irradiation of $365 \mathrm{~nm}$ (from left to right): $\mathrm{Eu}(\mathrm{III})$ cholate $(5 / 15 \mathrm{mM})$ doped with $6 \mathrm{mM}$ pyrene; $\mathrm{Tb}$ (III) cholate (5/15 mM), Tb(III)$\mathrm{Eu}(\mathrm{III})$ cholate (4.9/0.1/15 mM) and $\mathrm{Tb}(\mathrm{III})-\mathrm{Eu}(\mathrm{III})$ cholate $(4 / 1 / 15 \mathrm{mM})$ all doped with $400 \mathrm{mM}$ DHN. Reproduced from ref. [25] by permission of The Royal Society of Chemistry.

a low boiling solvent like hexane and dropcasted on the glass slides. After evaporation of the solvent the drop cast layers were examined under a handheld long wave UV lamp (Fig. 17). These films showed similar luminescent behaviour as observed for the xerogels. ${ }^{[25]}$ Therefore, in going from the hydrogel to the xerogel to the coated powder the sensitization was retained.

Thus we were successful in self-assembling multiple components in a supramolecular gel matrix and we have showed the processability of emissive xerogels. This approach provides a simple way of making light emitting materials. Attempts are ongoing in our laboratory to make white light emitting materials.

\section{Enzyme Assay Based on Lanthanide Luminescence}

Sensing and assay of biomolecules like enzymes is an important task with real life applications. There are multiple methods for enzyme assays which include colorimetric and fluorometric techniques.[26] Since we have a gel system which is mainly composed of biocompatible cholate and luminescent lanthanide

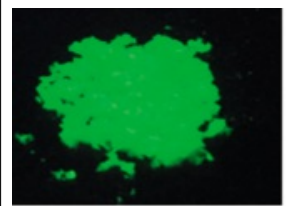

(a)

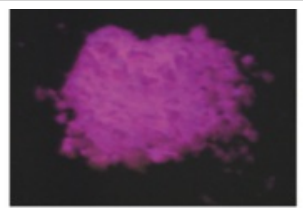

(b)

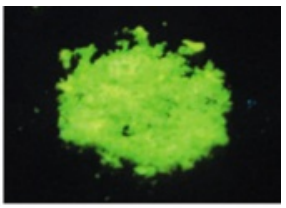

(C)

Fig. 16. Photographs of the xerogels (UV irradiation at $365 \mathrm{~nm}$ ) of (a) $\mathrm{Tb}$ (III) cholate (5/15 mM, doped with $400 \mathrm{mM} \mathrm{DHN})$, (b) Eu(III) cholate (5/15 $\mathrm{mM}$, doped with $6 \mathrm{mM}$ pyrene) and (c) $\mathrm{Tb}(\mathrm{III})-\mathrm{Eu}(\mathrm{III})$ cholate $(4 / 1 / 15 \mathrm{mM}$, doped with $400 \mathrm{mM}$ DHN). Reproduced from ref. [25] by permission of The Royal Society of Chemistry.

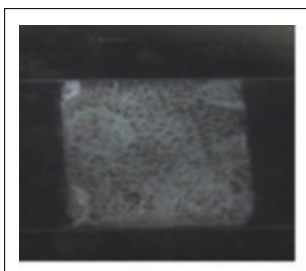

(a)

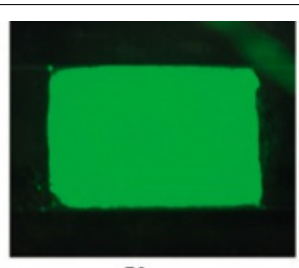

(b)

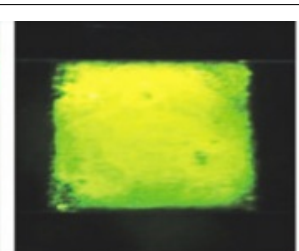

(c)

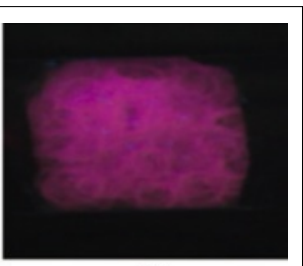

(d)

Fig. 17. Photographs of the thin films dropcasted from the suspensions of xerogels under UV irradiation at $365 \mathrm{~nm}$ of (a) Tb(II) cholate $(5 / 15 \mathrm{mM})$; (b) Tb(III) cholate (5/15 mM) + $400 \mathrm{mM} \mathrm{DHN}$; (c) $\mathrm{Tb}$ (III)-Eu(III) cholate (4/1/15 mM) $+400 \mathrm{~m} \mathrm{MDHN}$ and (d) Eu(III) cholate $(5 / 15 \mathrm{mM})+6 \mathrm{mM}$ pyrene. Reproduced from ref. [25] by permission of The Royal Society of Chemistry.

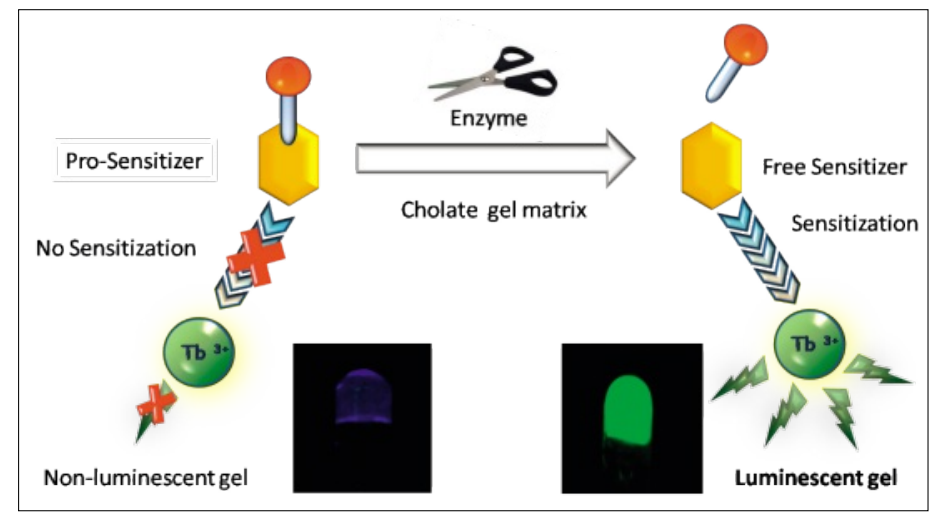

Fig. 18. A strategy towards the design of enzyme-induced luminescent gel. Reproduced from ref. [29] by permission of The Royal Society of Chemistry.

ions, we thought of utilizing this gel system for the assay of different classes of enzymes. Lanthanide luminescence has been utilized earlier in enzyme assays where the direct coordination of lanthanide ion with a suitable ligand was required.[27] We have used the sensitization of $\mathrm{Tb}(\mathrm{III})$ by $\mathrm{DHN}$ to develop a novel 'pro-sensitizer'-based assay of enzymes. This strategy is shown in Fig. 18.

Our idea was to incorporate the prosensitizer into the gel system which upon the action of an enzyme would release the sensitizer and thus enhance the $\mathrm{Tb}$ (III) luminescence. A simple diester of DHN was made which was susceptible for cleavage by the action of any esterase to release DHN, the sensitizer (Scheme 1). Since the malfunctioning of lipases causes several cardiac and liver diseases, ${ }^{[28]}$ a lipase was tested with pro-sensitizer 4.

Compound $\mathbf{4}$ did not show appreciable sensitization of $\mathrm{Tb}$ (III). But when lipase from Candida rugosa sp. was added significant enhancement of $\mathrm{Tb}$ (III) emission took place suggesting the release of DHN. A control experiment with denatured lipase did not show any appreciable enhancement of $\mathrm{Tb}$ (III) luminescence which further confirmed the enzyme activity in the earlier case (Fig. 19).

Our next attempt was to check the generality of this sensing system with other enzymes. $\beta$-Glucosidase was selected for this purpose, which cleaves water soluble glucosides to release sugars. Mono $\beta$-D

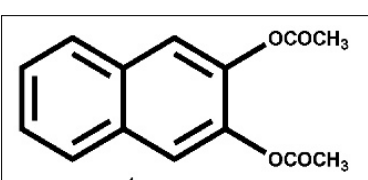

4
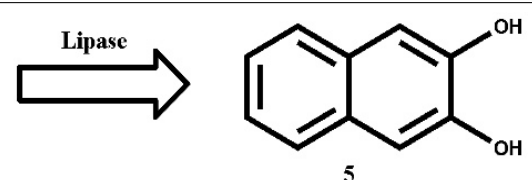

Scheme 1:

Generation of DHN due to the activity of lipase. 
glucoside of DHN was thus designed as another prosensitizer (Scheme 2).

This substrate also did not show appreciable sensitization of $\mathrm{Tb}(\mathrm{III})$, but in the presence of $\beta$-glucosidase it showed effective sensitization and green emission. This was attributed to the release of DHN by the action of the enzyme on prosensitizer 6 (Fig. 20). ${ }^{[29]}$

HPLC analysis of both gel systems (containing lipase and $\beta$-glucosidase) were carried out, which confirmed the release of DHN upon enzyme action. The HPLC profiles were in good agreement with the fluorescence measurements. Thus it was confirmed that the luminescence enhancement is only due to the release of DHN by the appropriate enzymes.

Thus, based on our lanthanide cholate gel systems we have developed assay techniques for two different classes of enzymes. Efforts towards developing assay techniques for various other enzymes are currently under progress in our laboratory.

\section{Conclusions and Future Outlook}

We have demonstrated that the facially amphiphilic bile acid backbone provides a powerful building block for designing a variety of hydrogelators. While the mechanism for the hydrogel formation remains to be well-understood, the primary driving force for the aggregation must be hydrophobic in nature. The hydrophobic pockets formed in the self-assembled fibrillar networks (SAFINs) accommodate a variety of molecular and nano-sized objects, allowing us to develop hybrid hydrogels. A self-assembly based approach was used to design a number of novel luminescent metallo hydrogels. This phenomenon was exploited to develop a novel 'pro-sensitizer' based enzyme assay. The stable network of calcium cholate hydrogels was utilized for creating silver and gold nanoparticles and a variety of metal sulfide nanoparticles embedded in the gel matrix.

We believe that this very general and simple strategy for the creation of metallo hydrogels will enable us to develop a variety of metal-derived nanoparticles for futuristic applications. The straightforward and rapid enzyme sensing can also lead to the development of enzyme sensing/assay kits for practical applications. Some of these possibilities are actively being pursued in the author's laboratory and these results will be published elsewhere in due course.

\section{Acknowledgments}

The work described in this account was generously funded by the Department of Science \& Technology, the Council of Scientific and Industrial Research, the Indo-French Centre for the Promotion of Advanced Research, New

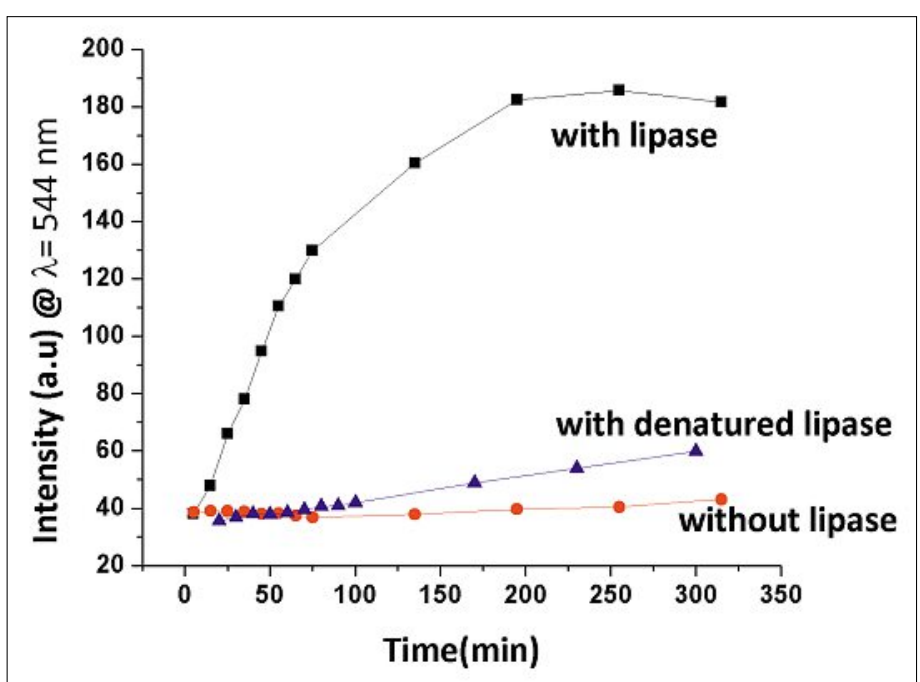

Fig. 19. Lipase (0.9 $\left.\mathrm{mg} \mathrm{mL}^{-1}\right)$ assay with $4(33 \mathrm{mM})$ in a Tb: cholate gel system (5 mM : $15 \mathrm{mM}$ ) at $298 \mathrm{~K}$; increase in intensity ( $\lambda 544 \mathrm{~nm})$ as a function of time for native enzyme and comparison with denatured enzyme or without any enzyme. Reproduced from ref. [29] by permission of The Royal Society of Chemistry.

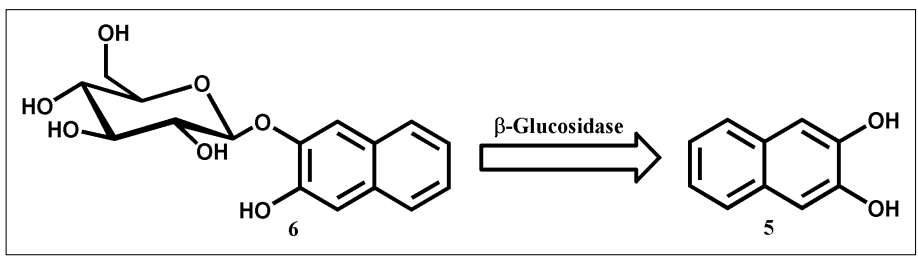

Scheme 2:

Generation of DHN by the activity of $\beta$-glucosidase.

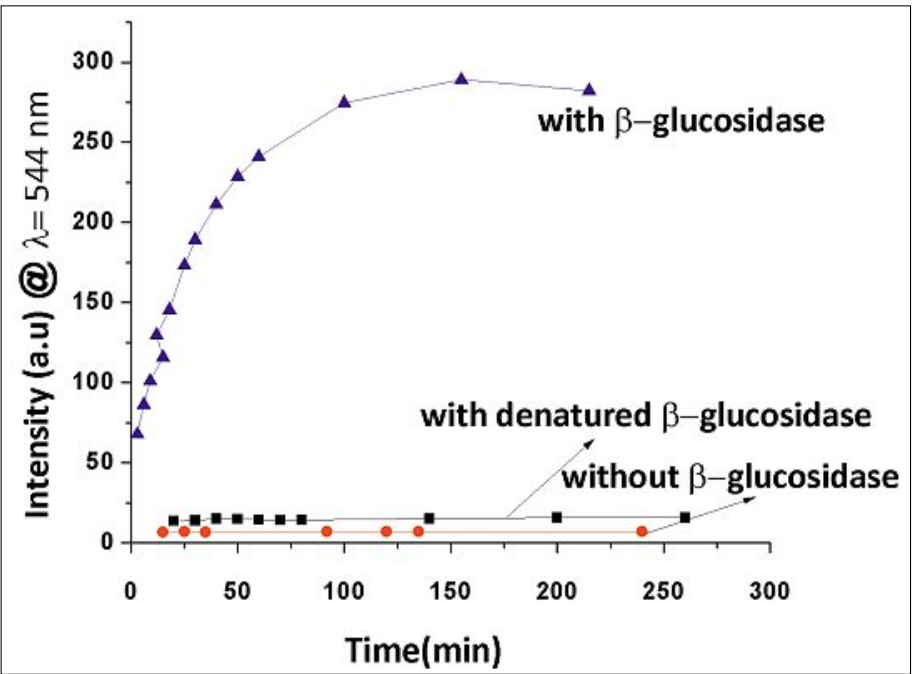

Fig. 20.

$\beta$-Glucosidase $(0.7$ $\mathrm{mg} \mathrm{mL}^{-1}$ ) assay with $6(0.37 \mathrm{mM})$ in a Tb: cholate gel system (5 mM : $15 \mathrm{mM}$ ) at $298 \mathrm{~K}$; increase in intensity (I $544 \mathrm{~nm}$ ) as a function of time for native $\beta$-glucosidase and comparison with denatured enzyme or without any enzyme. Reproduced from ref. [29] by permission of The Royal Society of Chemistry.

Delhi. We also thank the DST and the UGC for the awards of a JC Bose Fellowship to UM, and a D.S. Kothari Postdoctoral fellowship to SVS, respectively. This work would not have been possible without the active participation of a number of coworkers and collaborators, and their contributions are acknowledged through appropriate citations.

Received: November 8, 2012

[1] a) 'Supramolecular chemistry and selfassembly', special issue in Science 2002, 295; b) G. R. Whittell, M. D. Hager, U. S. Schubert, I. Manners, Nature Mater. 2011, 10, 176; c) T. Aida, E. W. Meijer, S. I. Stupp, Science 2012, 335,813

[2] a) K. Y. Lee, D. J. Mooney, Chem. Rev. 2001, 101, 1869; b) T. Miyata, T. Uragami, K. Nakame, Adv. Drug Delivery Rev. 2002, 54, 79; c) S. Banerjee, R. K. Das, U. Maitra, J. Mater. Chem. 2009, 19, 6649; d) A. Dawn, T. Shiraki, S. Haraguchi, S.-i. Tamaru, S. Shinkai,
Chem.-Asian J. 2011, 6, 266; e) K. Ramesh, S. Bhowmik, U. Maitra, J. Ind. Chem. Soc. 2011, 88, 1903.

[3] N. M. Sangeetha, U. Maitra, Chem. Soc. Rev $\mathbf{2 0 0 5}, 34,821$.

[4] a) U. Maitra, S. Nath, Chem.-Asian J. 2009 , 4, 989; b) S. Ghosh, A. R. Choudhaury, T. N Gururow, U. Maitra, Organic Lett. 2005, 7 , 1441; c) U. Maitra, S. Nath, ARKIVOC 2005, iii, 133 .

[5] N. Vijayalakshmi, U. Maitra, Organic Lett. 2005, 7, 2727.

[6] T. B. N. Satyanarayana, U. Maitra, A. J. Savyasachi, Eur. J. Org. Chem. 2012, 19, 3658.

[7] T. B. N. Satyanarayana, U. Maitra, Chem.Asian J. 2012, 7, 321.

[8] a) U. Maitra, S. Mukhopadhyay, A. Sarkar, P. Rao, S. S. Indi, Angew. Chem. Int. Ed. 2001, 40, 2281; b) S. Mukhopadhyay, G. Krishnamoorthy, U. Maitra, J. Phys. Chem. B. 2003, 107, 2189; c) S. Mukhopadhyay, U. Maitra, G Krishnamoorthy, J. Schmidt, Y. Talmon, J. Am. Chem. Soc. 2004, 126, 15905.

[9] P. Babu, D. Chopra, T. N. Guru Row, U. Maitra, Org. Biomol. Chem. 2005, 3, 3695. 
[10] a) M.-C. Daniel, D. Astruc, Chem. Rev. 2004 104, 293; b) E. Bisselier, D. Astruc, Chem. Soc. Rev. 2009, 38, 1759; c) L. Vigderman, B. P. Khanal, E. R. Zubarev, Adv. Mater. 2012, 24, 4811.

[11] S. Bhat, U. Maitra, Chem. Mater. 2006, 18, 4224.

[12] a) S. B. Schryver, Proc. R. Soc. London, Ser. B 1913, 86, 460; b) S. B. Schryver, Proc. R. Soc. London, Ser. B 1914, 87, 366; c) S. B. Schryver, Proc. R. Soc. London, Ser. B 1916, 89, 176; d) S. B. Schryver, Proc. R. Soc. London, Ser. B 1916 , 89, 361.

[13] Y. Qiao, Y. Lin, Y. Wang, Z. Yang, J. Liu, J. Zhou, Y. Yan, J. Huang, Nano Lett. 2009, 9, 4500 .

[14] A. Chakrabarty, U. Maitra, A.D. Das, J. Mater. Chem. 2012, 22, 18268.

[15] K. Binnemans, Chem. Rev. 2009, 109, 4283.

[16] a) K. Hanaoka, K. Kikuchi, H. Kojima, Y. Urano, T. Nagano, Angew. Chem., Int. Ed. 2003, 42, 2996; b) S. Mizukami, K. Tonai, M. Kaneko, K. Kikuchi, J. Am. Chem. Soc. 2008, 130, 14376.
[17] a) J. R. Perez, F. Nome, J. H. Fendler, J. Am Chem. Soc. 1977, 99, 7749; b) G. D. Correll, R. N. Cheser, F. Nome, J. H. Fendler, J. Am. Chem. Soc. 1978, 100, 1254; c) C. S. Bonnet, L. Pellegatti, F. Buron, C. M. Shade, S. Villette, V. Kubíček, G. Guillaumet, F. Suzenet, S. Petoud, E. Tóth, Chem. Commun. 2010, 46, 124.

[18] a) J.-C. G. Bünzli, Acc. Chem. Res. 2006, 39 , 53; b) S. V. Eliseva, J.-C. G. Bünzli, Chem. Soc. Rev. 2010, 39, 189.

[19] G. De Paoli, Z. Džolic, F. Rizzo, L. De Cola, F. Vögtle, W. M. Müller, G. Richardt, M. Žinic, Adv. Funct. Mater. 2007, 17, 821.

[20] S. Bhowmik, S. Banerjee, U. Maitra, Chem. Commun. 2010, 46, 8642 .

[21] H. G. Brittain, Inorg. Chem. 1978, 17, 2762.

[22] a) S. V. Eliseeva, D. N. Pleshkov, K. A Lyssenko, L. S. Lepnev, J.-C. G. Bünzli, N. P. Kuzmina, Inorg. Chem. 2010, 49, 9300; b) N. Kerbellec, D. Kustaryono, V. Haquin, M. Etienne, C. Daiguebonne, O. Guillou, Inorg. Chem. 2009, 48, 2837.

[23] J. M. de Souza, S. Alves, G. F. De Sa, W. M. de Azevedo, J. Alloys Compd. 2002, 344, 320.
[24] Y. Tsukahara, M. Sato, S. Katagiri, T. Honda, K. Nakamura, Y. Wada, J. Alloys Compd. 2008, 451, 194.

[25] S. Banerjee, R. Kandanelli, S. Bhowmik, U. Maitra, Soft Matter 2011, 7, 8207.

[26] a) G. Ghale, V. Ramalingam, A. R. Urbach, W. M. Nau, J. Am. Chem. Soc. 2011, 133, 7528; b) V. Sharma, R. S. Agnes, D. S. Lawrence, J. Am. Chem. Soc. 2007, 129, 2742.

[27] a) M. Halim, M. S. Tremblay, S. Jockusch, N. J. Turro, D. Sames, J. Am. Chem. Soc. 2007, 129, 770; b) R. A. Evangelista, A. Pollak, E. F. G. Templeton, Anal. Biochem. 1999, 197, 213; c) T. Steinkamp, U. Karst, Anal. Biochem. 2004, 380, 24.

[28] a) J. M. Littlewood, S. P. Wolfe, S. P. Conway, Pediatr. Pulmonol. 2006, 41, 35; b) A. T. Szymanska, J. Rujner, A. Lugowska, D. S. Korszynska, B. Wozniewicz, E. Czarnowska, Pediatr. Int. 2006, 6, 643.

[29] S. Bhowmik, U. Maitra, Chem. Commun. 2012 , $48,4624$. 